\title{
Direct optical entry through Palmer's point: a new technique for those at risk of entry-related trauma at laparoscopy
}

\author{
Thomas R. Aust • Salma I. Kayani • \\ David J. Rowlands
}

Received: 26 April 2009/Accepted: 4 June 2009/Published online: 24 June 2009

(C) Springer-Verlag 2009

\begin{abstract}
We present a technique of direct optical entry through Palmer's point $(3 \mathrm{~cm}$ below the left costal margin in the mid-clavicular line) as an alternative laparoscopic entry method for those gynaecological patients at risk of periumbilical adhesions. This method has been used in 15 patients and allowed the adhesions to be cleared where necessary. We feel that this technique can provide a safe approach to the abdominal cavity and should be considered as another method alongside closed and open umbilical techniques in the laparoscopic gynaecologist's repertoire.
\end{abstract}

Keywords Laparoscopic entry · Adhesions · Palmer's point · Optical entry

\section{Introduction}

Patients with periumbilical adhesions are at risk of bowel injury at the time of primary trocar insertion. The recently published Royal College of Obstetricians and Gynaecologists (RCOG) green-top guideline [1] on the prevention of entry-related injuries suggests that Palmer's point $(3 \mathrm{~cm}$

T. R. Aust $(\bowtie) \cdot$ D. J. Rowlands

Department of Obstetrics and Gynaecology,

Wirral University Teaching Hospital NHS Foundation Trust,

Arrowe Park Hospital,

Arrowe Park Road, Upton, Wirral,

Merseyside CH49 5PE, UK

e-mail: tomaust@doctors.org.uk

S. I. Kayani

University College Hospital,

235 Euston Road,

London NW1 2BU, UK below the left costal margin in the mid-clavicular line) is the safest alternative entry site [2]. We describe a new technique using direct optical entry at this site which then allows visual inspection of the umbilicus and (if necessary) adhesiolysis to allow subsequent insertion of an umbilical port under direct vision.

\section{Materials and methods}

Patient selection

Patients listed for diagnostic or operative laparoscopy with a history of one or more of the following: midline laparotomy, previous two or more transverse lower abdominal incisions, previous bowel surgery, presence of umbilical hernia, umbilical surgery or previously identified periumbilical adhesions.

Patients were excluded if they had splenomegaly, hepatomegaly or previous left upper quadrant surgery.

\section{Technique}

The stomach was emptied of secretions and air following endotracheal intubation. (This is most easily performed using an orogastric tube by the anaesthetist and is probably less traumatic than a nasogastric tube.)

The left upper quadrant was inspected for scars and the upper abdomen palpated for hepatomegaly or splenomegaly. A 5-mm incision was made over Palmer's point.

A 5-mm optical port (Endopath ${ }^{\circledR}$ XCEL $^{\mathrm{TM}}$, Ethicon, Bracknell, UK) was held vertically and the layers observed via a 5-mm laparoscope. A gentle rotating action in a vertical direction was used to allow the bladeless tip to separate the tissues. 
The layers of the abdominal wall seen at Palmer's point are as follows:
skin,
subcutaneous fat, external oblique aponeurosis, internal oblique aponeurosis, transversalis muscle fibres, (sometimes) extraperitoneal fat, peritoneum.

Once the peritoneum was breached the laparoscope and introducer were carefully removed from the port. The laparoscope was then reintroduced as insufflation was commenced at high flow (maximum of $10 \mathrm{~L} / \mathrm{min}$ ) until a pressure of $20 \mathrm{mmHg}$ was achieved.

An extra $360^{\circ}$ check was then performed to exclude a through-and-through bowel injury. The umbilicus was then inspected and any adhesions cleared using one or more 5-mm ports inserted under direct vision. The rest of the laparoscopy was continued once a $10-\mathrm{mm}$ umbilical port could be safely placed. Once all ports were inserted, the pressure was reduced to $15 \mathrm{mmHg}$.

At the end of the operation, the skin was closed using a single subcuticular suture.

\section{Results}

We have performed this technique for 15 patients so far. The ages ranged from 28 to 70 years and body mass index (BMI) from 21 to 48 . There were no entry-related complications and periumbilical adhesions seen in 12 of the 15 patients.

\section{Discussion}

Complications associated with entry at laparoscopic surgery, such as bowel or vascular damage, are fortunately rare $[3,4]$.

In most patients, the base of the umbilicus offers the best point of entry to the abdomen as the abdominal wall is thinnest at that point. However, in patients with a history of previous surgery, the risks of periumbilical adhesions increase. In their prospective single-centre study of 814 patients, Audebert and Gomel [5] found a progressive increase in the incidence of adhesions at the umbilicus as follows: least in those with no prior surgery $(0.68 \%)$, then those with a previous laparoscopy (1.6\%), then those with a previous horizontal suprapubic incision $(19.8 \%)$ and most in those with a previous midline incision $(51.7 \%)$. It then follows that these factors could increase the risk of bowel damage at blind entry through the umbilicus.

Hasson described an open entry technique as he felt "uncomfortable about inserting needles and sharp trocars blindly into the abdomen" [6]. Although proponents of this technique feel that open entry could reduce the incidence of complications such as vessel injury, it is hard to see how a piece of adherent bowel would be less likely to be injured during an open entry than closed. The RCOG guideline states that most studies show no significant advantage of the open technique over closed [1].

Palmer's point entry using initial Veress needle insufflation has been reported by a number of authors in the form of case series [7-9]. In a randomised study, Azevedo and colleagues [10] compared intraumbilical and left hypochondrium Veress entry. They found similar rates of failure of placement of the needle in the peritoneal cavity using saline drop, aspiration and pressure/flow tests. It could be argued that the correct placement of the Veress needle would be more difficult in the left upper quadrant as the fascial layers of the abdomen do not come together as they do at the umbilicus. This potential problem may be overcome by the use of an optical entry trocar.

The use of an optical entry trocar has been pioneered by general surgeons. Problems with gas leaks around the port (with open entry) and the risk of inadvertent injury (with closed entry) were given as reasons for using an optical entry in a paper of 650 surgical patients [11]. Through a $15-\mathrm{mm}$ incision, they mainly used the umbilical site for initial entry but also reported entry at sites in all four quadrants of the abdomen. Berch et al. [12] report using an optical entry $12-\mathrm{mm}$ port in the left upper quadrant for their series of 370 patients undergoing laparoscopic gastric bypass procedures. The mean BMI was 49.7 and over half had had previous abdominal procedures. They reported very low incidences of visceral or vessel damage, abdominal wall bleeding and port-site hernias and use this technique as their standard approach.

To our knowledge, this is the first report of a case series using a direct optical entry technique using Palmer's point in gynaecological patients. We used a $5-\mathrm{mm}$ port rather than the $12-15 \mathrm{~mm}$ reported by general surgeons; this was mainly to avoid the need for fascial closure and to improve cosmesis. In a few cases, we continued to use the initial 5-mm laparoscope for the remainder of the operation, but in most cases, the Palmer's point entry simply allowed safe insertion of the $10-\mathrm{mm}$ umbilical port through which a wider laparoscope was inserted. This allowed for an improved view of the pelvis that the general surgeons probably did not require.

As the incidence of periumbilical adhesions and, therefore, bowel-related injury is low in most patients, this technique can be reserved for those with risk factors such as previous surgery or paraumbilical hernias. The new RCOG guidelines [1] also suggest that alternative entry sites be considered for very thin or morbidly obese patients so our method may also help in those circumstances. We feel that 
this technique can provide an alternative approach to the abdominal cavity and should be considered as another method alongside closed and open umbilical techniques in the laparoscopic gynaecologist's repertoire.

\section{Acknowledgements None.}

Disclosure of interests I can confirm that there are no conflicts of interest for any of the authors.

Contribution to authorship TRA wrote the paper, SIK collated and analysed the data and DJR conceived the technique and carried out the procedures.

Details of ethics approval Ethical approval was not sought as this technique is a modification of Palmer's entry which is a recognised method of laparoscopic entry.

Funding There was no funding for this study.

\section{References}

1. RCOG (2008) Preventing entry-related gynaecological laparoscopic injuries; Green-Top Guideline No. 49. RCOG, London

2. Palmer R (1974) Safety in laparoscopy. J Reprod Med 13(1):1-5
3. Garry R (1997) Complications of laparoscopic entry. Gynecol Endose 6:319-329

4. Jansen FW, Kapiteyn K, Trimbos-Kemper T, Hermans J, Trimbos JB (1997) Complications of laparoscopy: a prospective multicentre observational study. Br J Obstet Gynaecol 104(5):595-600

5. Audebert AJ, Gomel V (2000) Role of microlaparoscopy in the diagnosis of peritoneal and visceral adhesions and in the prevention of bowel injury associated with blind trocar insertion. Fertil Steril 73(3):631-635

6. Hasson HM (1974) Open laparoscopy: a report of 150 cases. J Reprod Med 12(6):234-238

7. Agarwala N, Liu CY (2005) Safe entry techniques during laparoscopy: left upper quadrant entry using the ninth intercostal space - a review of 918 procedures. J Minim Invasive Gynecol 12 (1):55-61

8. Childers JM, Brzechffa PR, Surwit EA (1993) Laparoscopy using the left upper quadrant as the primary trocar site. Gynecol Oncol 50(2):221-225

9. Lam KW, Pun TC (2002) Left upper quadrant approach in gynecologic laparoscopic surgery using reusable instruments. J Am Assoc Gynecol Laparosc 9(2):199-203

10. Azevedo OC, Azevedo JL, Sorbello AA, Miguel GP, Guindalini RS, Godoy AC (2006) Veress needle insertion in the left hypochondrium in creation of the pneumoperitoneum. Acta Cir Bras 21(5):296-303

11. String A, Berber E, Foroutani A, Macho JR, Pearl JM, Siperstein AE (2001) Use of the optical access trocar for safe and rapid entry in various laparoscopic procedures. Surg Endosc 15(6):570-573

12. Berch BR, Torquati A, Lutfi RE, Richards WO (2006) Experience with the optical access trocar for safe and rapid entry in the performance of laparoscopic gastric bypass. Surg Endosc 20 (8):1238-1241 